\title{
Linear Baseband Precoding Strategies for Millimeter Wave MIMO Multi-X Channels
}

\author{
Venkatesh Ramireddy, Marcus Grossmann, Markus Landmann, Rudolf Zetik and Giovanni Del Galdo \\ Fraunhofer Institute for Integrated Circuits IIS, D-98693 Ilmenau, Germany \\ \{venkatry, marcus.grossmann, markus.landmann, rudolf.zetik, giovanni.delgaldo\} @iis.fraunhofer.de
}

\begin{abstract}
This paper focuses on the design of linear baseband precoding and combining techniques for millimeter wave (mmWave) multiple input multiple output (MIMO) backhaul networks. We consider a scenario known as MIMO multi-X channel with $L$ transmitter base stations and $K$ receiver base stations, each equipped with multiple antennas, where each transmitter base station communicates simultaneously with all receiver base stations. The extremely short wavelength of mmWave requires the use of large antenna arrays at the base stations (BS) to combat the high path loss to achieve sufficiently high link gains, enabled by directional beamforming. As signal processing with large antenna arrays lead to implementations that are associated with high power consumption and high hardware complexity, hybrid (analog/digital) precoding/combining has been proposed recently to overcome these limitations. The design of such hybrid precoders/combiners is typically performed separately for the analog and the digital domains. In this work, we propose several novel low-complexity schemes suited for the digital part of such hybrid precoders/combiners. Our solutions are based on blockdiagonalization and minimum mean squared error (MMSE) techniques and assume the availability of partial knowledge of CSI at each BS in the network. Finally the simulation results demonstrate the potential gains of the proposed algorithms.
\end{abstract}

Index Terms-MIMO multi-X, hybrid precoding, RF chains, block diagonalization, MMSE, computational complexity, wireless backhaul

\section{INTRODUCTION}

The underutilized spectrum in the millimeter wave (mmWave) bands can be exploited in order to meet the interminable demands for high data rates. Especially in the context of future $5 \mathrm{G}$ communication systems, the backhaul has to cope up with huge amounts of handling data to provide high speed access to the users. Due to the small physical size of the antenna elements at extremely short wavelengths, mmWave systems can be employed with large-scale phased arrays with beam steering capabilities to enable high gain cellular backhaul links to combat the high path loss [1] at $\mathrm{GHz}$ frequencies. However, for such large-scale arrays the conventional digital baseband (BB) precoding (combining) at the transmitter (receiver) may not always be feasible due to the very high hardware costs and power consumption of the array. Hence, a possible solution could be to use hybrid (analog-digital) precoding/combining where initially the transmit/received signals are combined with a less dimensional radio frequency $(\mathrm{RF})$ chains before further processing them in the digital domain.

Hybrid precoding for the single user multiple input multiple output (SU-MIMO) was initially proposed in [2]. By assuming the mmWave channel as sparse in the angular domain, the authors in [2] computed the hybrid ( $\mathrm{RF}$ and $\mathrm{BB}$ ) precoders jointly using the orthogonal matching pursuit (OMP) algorithm. Hybrid precoding for multi-user MIMO (MU-MIMO) was proposed in [3], [4] and [5]. In [3], the mean squared error (MSE) criterion was used to jointly design the RF and BB precoders, respectively using the OMP algorithm. The authors in [4], [5] used the two step approach to compute the hybrid precoders. In the first step, the RF precoders and combiners are computed to maximize the energy in the respective channel, while the interference is aligned in the second step by computing the BB precoders and combiners on the effective channel resulting after RF precoding and combining. For the first time, the authors in [6], proposed the joint RF and BB precoder design using the MSE based criterion in MIMO interference channels. In this paper, we consider the MIMO multi-X channel, where each transmit base station (TBS) transmits multiple data streams to each receive base station (RBS) simultaneously as shown in Fig. 1. Unlike the MIMO interference channel, where each TBS has intended data to a single RBS, the TBS in the MIMO multi-X channel has data intended to $K$ RBSs. Hence, each RBS suffers from a high degree of interference. Recently, it has been considered that the dense deployment of multiple base stations (BS) with reduced hardware requirements is a potential candidate for future $5 \mathrm{G}$ networks to realize thousand-fold increase in system capacities. Hence design of hybrid precoders and combiners for such MIMO multi-X channels has become a very important research area.

In general, the RF and BB precoders are computed separately due to the high complexity involved in the joint design. However, for such channels, the design of RF precoders and combiners is still an open question and an optimal solution is not yet known due to the constraints placed on its entries. However, the maximum achievable sum rate in the case of hybrid precoding is bounded by the sum rate achieved by the BB only precoding [2], [3], [6], we only consider the BB precoding which can be applied directly on the effective channel resulting after the RF precoding and combining. Since we consider the objective of maximizing the sum rate, the optimal approach to design the $\mathrm{BB}$ precoders and combiners that maximizes the sum rate leads to an optimization problem that has a very high computational complexity. Therefore, in this paper we propose several low-complexity design schemes based on the well known block-diagonalization (BD) algorithm 


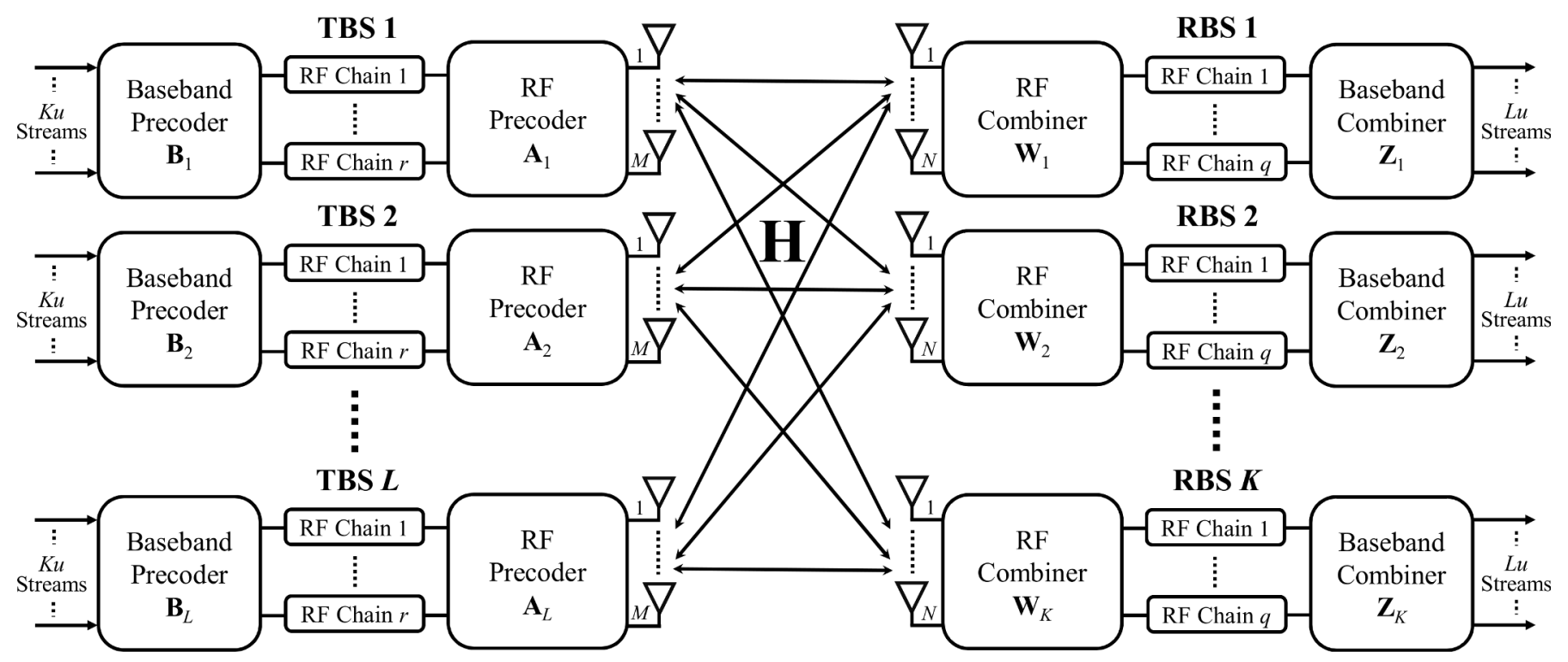

Fig. 1. A wireless hybrid backhaul network with $L$ TBSs and $K$ RBSs, where each TBS communicates simultaneously with all RBSs.

[8] and minimum mean squared error (MMSE) criterion. As we shall show in the subsequent sections, the direct application of $\mathrm{BD}$ is not straight forward for MIMO multi-X channel due to the different types of interference present in the MIMO multi-X channel. Moreover, only partial channel state information (CSI) is assumed to be available at each BS. The number of RF chains employed at each TBS and RBS plays a crucial role in determining the multiplexing gain of the system. In order to achieve the highest multiplexing gain, the relation between the number of transmit RF chains and receive RF chains is also derived.

The following notations are used throughout the paper. $\mathbf{C}^{H}$, $\mathbf{C}^{T}, \mathbf{C}^{-1}$ and $\|C\|_{F}$ are the hermitian transpose, transpose, inverse and frobenius norm of $\mathbf{C}$, respectively. $\mathbf{I}_{n}$ is an identity matrix of size $n$. The block diagonal matrix with matrix entries on the main diagonal is represented by blkdiag. $\mathcal{C N}(m, \mathbf{R})$ denotes the complex Gaussian random vector with mean $m$ and covariance matrix $\mathbf{R}$.

The remainder of the paper is organized as follows. The system model for transmission over the mmWave MIMO multi-X channel is described in Section II. The design of the BB precoding and combining schemes is explained in Section III. In Section IV, we present the relation between the number of RF chains required at each TBS and RBS to achieve the highest multiplexing gain. The computational complexity of the proposed schemes is analyzed in Section V, and the simulation results are presented in Section VI. Finally, Section VII concludes the paper.

\section{SySTEM MODEL AND PROBLEM STATEMENT}

\section{A. System Model}

Consider the wireless hybrid backhaul network consisting of $L$ TBSs and $K$ RBSs shown in Fig. 1. Each TBS is equipped with $M$ antennas, $r$ RF chains and transmits $K u$ data streams to serve $K$ RBSs, which are equipped with $N$ receive antennas and $q$ RF chains each. The signal transmitted at the $l$ th TBS can be expressed as $\boldsymbol{x}_{l}=\mathbf{A}_{l} \mathbf{B}_{l} \boldsymbol{s}_{l} \in \mathbb{C}^{M \times 1}$, where $\mathbf{A}_{l} \in$ $\mathbb{C}^{M \times r}$ is the RF precoder, implemented using analog phase shifters,

$$
\mathbf{B}_{l}=\left[\mathbf{B}_{1, l}, \mathbf{B}_{2, l}, \ldots, \mathbf{B}_{K, l}\right]
$$

is the digital $\mathrm{BB}$ precoder of size $r \times K u$, and $\boldsymbol{s}_{l}=$ $\left[\boldsymbol{s}_{1, l}^{T}, \boldsymbol{s}_{2, l}^{T}, \ldots, \boldsymbol{s}_{K, l}^{T}\right]^{T} \in \mathbb{C}^{K u \times 1}$ is the data symbol vector with $\mathbb{E}\left\{\boldsymbol{s}_{l} \boldsymbol{s}_{l}^{H}\right\}=\mathbf{I}_{K u}$. Here, $\boldsymbol{s}_{k, l}$ contains the $u$ data symbols associated to TBS $l$ and RBS $k$. In addition, the power constraint $\left\|\mathbf{A}_{l} \mathbf{B}_{l}\right\|_{F}^{2} \leq P_{l}$ is enforced at the $l$ th TBS with $P_{l}$ being the total transmission power.

Adopting a narrowband block-fading channel model, the received signal at the $k$ th $\mathrm{RBS}$ is given by

$$
\boldsymbol{y}_{k}=\sum_{l=1}^{L} \mathbf{H}_{k, l} \mathbf{A}_{l} \mathbf{B}_{l} \boldsymbol{s}_{l}+\boldsymbol{n}_{k}=\mathbf{H}_{k} \mathbf{A B} \boldsymbol{s}+\boldsymbol{n}_{k},
$$

where $\mathbf{H}_{k}=\left[\mathbf{H}_{k, 1}^{T}, \mathbf{H}_{k, 2}^{T}, \ldots, \mathbf{H}_{k, L}^{T}\right]^{T} \in \mathbb{C}^{N \times L M}$ with $\mathbf{H}_{k, l}$ being the $N \times M$ channel matrix between the $k$ th RBS and the $l$ th TBS and $\boldsymbol{n}_{k} \sim \mathcal{C N}\left(0, \sigma_{0}^{2}\right)$ is the i.i.d. additive white Gaussian noise. At the $k$ th RBS, the BB and RF precoders $\mathbf{Z}_{k} \in \mathbb{C}^{q \times L u}$ and $\mathbf{W}_{k} \in \mathbb{C}^{N \times q}$, respectively, are used to process the received signal vector $\boldsymbol{r}_{k}$ as

$$
\begin{aligned}
\boldsymbol{r}_{k} & =\mathbf{Z}_{k}^{H} \mathbf{W}_{k}^{H} \boldsymbol{y}_{k}=\mathbf{Z}_{k}^{H} \mathbf{W}_{k}^{H} \sum_{l=1}^{L} \mathbf{H}_{k, l} \mathbf{A}_{l} \mathbf{B}_{k, l} \boldsymbol{s}_{k, l} \\
& +\mathbf{Z}_{k}^{H} \mathbf{W}_{k}^{H} \sum_{l=1}^{L} \mathbf{H}_{k, l} \mathbf{A}_{l} \sum_{j \neq k, j=1}^{K} \mathbf{B}_{j, l} \boldsymbol{s}_{j, l}+\mathbf{Z}_{k}^{H} \mathbf{W}_{k}^{H} \boldsymbol{n}_{k}
\end{aligned}
$$


where the the RF combiner $\mathbf{W}_{k}$ has a similar structure as $\mathbf{A}_{l}$ with its entries being of constant modulus.

In this work, we are only concerned on the design of the BB precoders and combiners, and therefore, we define the effective channel that includes the RF precoding and combiners between the $l$ th TBS and $k$ th RBS as

$$
\mathbf{G}_{k, l}=\mathbf{W}_{k}^{H} \mathbf{H}_{k, l} \mathbf{A}_{l} \in \mathbb{C}^{q \times r}, \forall l, k,
$$

which is bounded by the number of RF chains such that $\|\mathbf{G}\|_{F}^{2}>\|\mathbf{H}\|_{F}^{2}$. Due to multipath propagation which results mainly due to reflections at mmWave frequencies, and no knowledge of RF precoding and combining at this stage, we design the precoders on the effective channel which is assumed to be full rank. However, the derivation in the case of rank deficient effective channels is straight forward. Based on (3) and (4), the output of the BB combiner can be written as

$$
\begin{aligned}
\boldsymbol{r}_{k, l}= & \underbrace{\mathbf{Z}_{k, l}^{H} \mathbf{G}_{k, l} \mathbf{B}_{k, l} \boldsymbol{s}_{k, l}}_{\begin{array}{c}
\text { desired signal with } \\
\text { inter-stream interference (ISI) }
\end{array}}+\underbrace{\mathbf{Z}_{k, l}^{H} \mathbf{G}_{k, l} \sum_{j \neq k, j=1}^{K} \mathbf{B}_{j, l} \boldsymbol{s}_{j, l}}_{\text {self interference (SI) }} \\
& +\underbrace{\mathbf{Z}_{k, l}^{H} \sum_{j \neq l, j=1}^{L} \mathbf{G}_{k, j} \sum_{i=1}^{K} \mathbf{B}_{i, j} \boldsymbol{s}_{i, j}}_{\text {multi-base station interference (MBI) }}+\underbrace{\mathbf{Z}_{k, l}^{H} \overline{\boldsymbol{n}}_{k}}_{\text {combined noise }} .
\end{aligned}
$$

\section{B. Problem statement}

The objective of our work is design the BB precoders at the TBSs and combiners at the RBSs to maximize the sum rate of the whole system. Assuming Gaussian signaling over the mmWave channel, the rate of link $(k, l)$ is given by

$$
R_{k, l}=\log \left(\left|\mathbf{I}_{u}+\frac{\mathbf{Z}_{k, l}^{H} \mathbf{G}_{k, l} \mathbf{B}_{k, l} \mathbf{B}_{k, l}^{H} \mathbf{G}_{k, l}^{H} \mathbf{Z}_{k, l}}{\mathbf{Z}_{k, l}^{H} \mathbf{X}_{k, l} \mathbf{X}_{k, l}^{H} \mathbf{Z}_{k, l}+\mathbf{Z}_{k, l}^{H} \mathbf{Z}_{k, l} \sigma_{0}^{2}}\right|\right),
$$

where

$$
\mathbf{X}_{k, l}=\mathbf{G}_{k, l} \sum_{j \neq k, j=1}^{K} \mathbf{B}_{j, l}+\sum_{j \neq l, j=1}^{L} \mathbf{G}_{k, j} \sum_{i=1}^{K} \mathbf{B}_{i, j} .
$$

Maximizing the sum rate $\sum_{(k, l)} R_{k, l}$ requires a joint optimization of all precoders and combiners. However, for similar optimization problems finding the global solution is computationally intractable [7]. To simplify the design, the joint precoder-combiner optimization can be decoupled, where the combiner matrices are computed, while the precoders are assumed to be fixed. The optimal combiners are then given by the well-known MMSE solution. The maximization of the sum rate can then be formulated as

$$
\begin{array}{ll}
\max _{\mathbf{B}_{l, k}, \forall l, k} & \sum_{l=1}^{L} \sum_{k=1}^{K} R_{k, l} \\
\text { subject to } & \left\|\mathbf{B}_{l}\right\|_{F}^{2} \leq P_{l}, \forall l .
\end{array}
$$

The above optimization problem is non-convex due to the non-convexity of the objective function. Therefore, finding the optimal solution is intractable. Hence, in this paper, we consider less computationally complex algorithms such as BD and MMSE for the design of the precoders and combiners.

\section{BASEBAND PRECODER COMBINER DESIGN}

From (5), it can be observed that three types of interferences, namely the inter-stream interference (ISI) (interference between the desired streams), self interference (SI) (intended information from TBS $l$ to other $\operatorname{RBS} j, \forall j \neq k, j=$ $1, \ldots, K)$ and the multi-base station interference (MBI) (intended signal to RBS $i \neq k$ from all TBS $\forall i \neq l, i=$ $1, \ldots, L)$ are present in the received signal. Hence, efficient $\mathrm{BB}$ precoding and combining schemes need to be devised appropriately to align the interferences. Moreover, since the design of $\mathrm{BB}$ precoders and combiners requires the knowledge of CSI at each base station, we assume that perfect CSI $\left[\mathbf{G}_{1, l}, \mathbf{G}_{2, l}, \ldots, \mathbf{G}_{K, l}\right]$ and $\left[\mathbf{G}_{k, 1}, \mathbf{G}_{k, 2}, \ldots, \mathbf{G}_{k, L}\right]$ is available at $l$ th TBS and $k$ th RBS, respectively.

\section{A. BD-BD scheme}

Due to the complex structure of the MIMO multi-X channel, the computation of $\mathrm{BB}$ precoders and combiners using conventional BD processing is not straightforward. This is due to the fact that at each base station (BS), only partial CSI is assumed to be available, but the conventional BD processing requires global CSI at each BS which is quite challenging. Hence, an appropriate combining scheme has to be devised initially to decouple the channels from $L$ TBSs to eliminate the MBI. In other words, the MIMO multi-X channel is decomposed to $L$ non-interfering MIMO broadcast (BC) sub-channels seen from each RBS, prior to applying the standard $\mathrm{BD}$ processing at each TBS. Therefore, we proceed by defining a two stage combiner

$$
\mathbf{Z}_{k}=\mathbf{T}_{k} \mathbf{Q}_{k}
$$

where $\mathbf{T}_{k}=\left[\mathbf{T}_{k, 1}, \mathbf{T}_{k, 2}, \ldots, \mathbf{T}_{k, L}\right] \in \mathbb{C}^{q \times L u}$ and $\mathbf{Q}_{k}=$ blkdiag $\left\{\mathbf{Q}_{k, 1}, \mathbf{Q}_{k, 2}, \ldots, \mathbf{Q}_{k, L}\right\} \in \mathbb{C}^{L u \times L u}$. To obtain $\mathbf{T}_{k}$, define a matrix at each $\operatorname{RBS} k$ as

$$
\begin{aligned}
& \overline{\mathbf{G}}_{k, i}=\left[\mathbf{G}_{k, 1}, \ldots, \mathbf{G}_{k, i-1}, \mathbf{G}_{k, i+1}, \ldots, \mathbf{G}_{k, L}\right] \\
& \forall i=1,2, \ldots, L .
\end{aligned}
$$

By applying the singular value decomposition (SVD), $\overline{\mathbf{G}}_{k, i} \in$ $\mathbb{C}^{q \times(L-1) r}$ can be decomposed as

$$
\overline{\mathbf{G}}_{k, i}=\left[\begin{array}{ll}
\overline{\mathbf{U}}_{k, i}^{\|} & \overline{\mathbf{U}}_{k, i}^{\perp}
\end{array}\right] \overline{\mathbf{\Sigma}}_{k, i} \overline{\mathbf{V}}_{k, i}^{H} .
$$

Here, $\overline{\mathbf{U}}_{k, i}^{\perp} \in \mathbb{C}^{q \times\left(q-\bar{g}_{k, i}\right)}$ comprises the last $\left(q-\bar{g}_{k, i}\right)$ left singular vectors that forms an orthogonal basis for the null space of $\overline{\mathbf{G}}_{k, i}$ with rank $\bar{g}_{k, i}=\min \{q,(L-1) r\}$. The null space when multiplied by $\overline{\mathbf{G}}_{k, i}$ nullifies the MBI. Therefore, the first stage of combiner is chosen as

$$
\mathbf{T}_{k, i}=\overline{\mathbf{U}}_{k, i}^{\perp} .
$$

After the first stage of combining, the resultant channel at $k$ th RBS can be expressed as

$$
\begin{aligned}
\mathbf{F}_{k} & =\mathbf{T}_{k}^{H} \mathbf{G}_{k} \in \mathbb{C}^{L\left(q-\bar{g}_{k, l}\right) \times L r} \\
& =\operatorname{blkdiag}\left\{\mathbf{F}_{k, 1}, \mathbf{F}_{k, 2}, \ldots, \mathbf{F}_{k, L}\right\}
\end{aligned}
$$


The matrices on the main diagonal denotes the resultant channels from $L$ TBSs to $k$ th RBS that are decoupled after the first stage of combining. Now, the resultant channel is fedback to the respective TBS to design the precoders that removes the remaining interference. Now, the design of precoders at each TBS $l$ follows by defining the matrix $\hat{\mathbf{F}}_{j, l}$ as

$$
\begin{array}{r}
\hat{\mathbf{F}}_{j, l}=\left[\mathbf{F}_{1, l}^{T}, \ldots, \mathbf{F}_{j-1, l}^{T}, \mathbf{F}_{j-1, l}^{T}, \ldots, \mathbf{F}_{K, l}^{T}\right]^{T}, \\
\forall j=1,2, \ldots, K .
\end{array}
$$

By performing SVD, $\hat{\mathbf{F}}_{j, l} \in \mathbb{C}^{(K-1)\left(q-\bar{g}_{j, l}\right) \times r}$ can be decomposed as

$$
\hat{\mathbf{F}}_{j, l}=\hat{\mathbf{U}}_{j, l}^{H} \hat{\boldsymbol{\Sigma}}_{j, l}\left[\begin{array}{ll}
\hat{\mathbf{V}}_{j, l}^{\|} & \hat{\mathbf{V}}_{j, l}^{\perp}
\end{array}\right]^{H} .
$$

Here, $\hat{\mathbf{V}}_{j, l}^{\perp} \in \mathbb{C}^{r \times\left(r-\hat{f}_{j, l}\right)}$ comprises the last $\left(r-\hat{f}_{j, l}\right)$ right singular vectors with rank $\hat{f}_{j, l}=\min \left\{(K-1)\left(q-\bar{g}_{k, i}\right), r\right\}$. Furthermore, define the SVD

$$
\mathbf{F}_{j, l} \mathbf{P}_{j, l}=\check{\mathbf{U}}_{j, l} \check{\Sigma}_{j, l} \check{\mathbf{V}}_{j, l}^{H} \in \mathbb{C}^{\left(q-\bar{g}_{j, l}\right) \times r}
$$

of the projection on to the null space of $\left[\hat{\mathbf{H}}_{l}\right]_{j}$, where $\mathbf{P}_{j, l}=$ $\hat{\mathbf{V}}_{j, l}^{\perp} \hat{\mathbf{V}}_{j, l}^{\perp H}$ is the projection matrix. The first $\left(r-\hat{f}_{j, l}\right)$ columns of $\tilde{\mathbf{V}}_{j, l}$ corresponds to the transmission vectors that eliminates the remaining interference. Hence, the columns of precoder $\mathbf{B}_{j, l}$ are chosen as

$$
\mathbf{B}_{j, l}=\left[\check{\mathbf{V}}_{j, l}\right]_{\left[:, 1: \hat{f}_{j, l}\right]} .
$$

In addition, the second stage of the combiner at $j$ th RBS is given by

$$
\mathbf{Q}_{j, l}^{\mathrm{BD}}=\left[\check{\mathbf{U}}_{j, l}\right]_{\left[:, 1: \hat{f}_{j, l}\right]} \cdot
$$

Using (8) the received signal at $k$ th RBS can be expressed as

$$
\boldsymbol{r}_{k}=\underbrace{\mathbf{Q}_{k}^{H} \mathbf{T}_{k}^{H} \mathbf{G}_{k} \mathbf{B}}_{\mathbf{C}_{k}} s+\mathbf{Q}_{k}^{H} \mathbf{T}_{k}^{H} \overline{\boldsymbol{n}}_{k},
$$

where $\mathbf{C}_{k}$ is a diagonal matrix. Hence, the design of precoders and combiners at each TBS and RBS, decouples the MIMO multi-X channel to $K L u$ parallel channels.

Similar performance can be achieved by choosing

$$
\mathbf{B}_{j, l}=\hat{\mathbf{V}}_{j, l}^{\perp}
$$

from (14) and computing $\mathbf{Q}_{k}$ using the MMSE solution that minimizes the mean squared error (MSE) which can be formulated as [9]

$$
\underset{\mathbf{Q}_{k, l}}{\arg \min } \mathbb{E}\left[\left\|\boldsymbol{s}_{k, l}-\mathbf{Q}_{k, l}^{H} \boldsymbol{r}_{k, l}\right\|_{2}^{2}\right] .
$$

By solving the above problem, the second stage of combiner $\mathbf{Q}_{k, l}$ can be given by

$$
\begin{aligned}
\mathbf{Q}_{k, l}^{H} & =\mathbb{E}\left[\hat{\boldsymbol{r}}_{k, l} \hat{\boldsymbol{r}}_{k, l}^{H}\right]^{-1} \mathbb{E}\left[\boldsymbol{s}_{k, l} \hat{\boldsymbol{r}}_{k, l}^{H}\right] \in \mathbb{C}^{u \times u} \\
& =\left(\mathbf{B}_{k, l}^{H} \mathbf{F}_{k, l}^{H}\right)\left(\mathbf{F}_{k, l} \mathbf{B}_{k, l} \mathbf{B}_{k, l}^{H} \mathbf{F}_{k, l}^{H}+\sigma_{0}^{2} \mathbf{I}_{k, l}\right)^{-1} .
\end{aligned}
$$

Hence, the precoders and the second stage of combiners can be computed simply by using (19) and (21).

\section{B. BD-MMSE scheme}

In this subsection, we propose BD-MMSE scheme that incorporates the idea of designing the precoders using $\mathrm{BD}$ [8] and the combiners using the MMSE [9] criterion. The $\mathrm{BD}$ processing at the TBSs decomposes the channel to $K$ non-interfering MIMO multiple access channels (MAC) subchannels seen from each TBS. In the first step, the precoders

(1) are computed as follows.

Define a matrix at $l$ th TBS as

$$
\begin{array}{r}
\tilde{\mathbf{G}}_{i, l}=\left[\mathbf{G}_{1, l}^{T}, \ldots, \mathbf{G}_{i-1, l}^{T}, \mathbf{G}_{i-1, l}^{T}, \ldots, \mathbf{G}_{K, l}^{T}\right]^{T}, \\
i=1,2, \ldots, K .
\end{array}
$$

The SVD of $\tilde{\mathbf{G}}_{i, l} \in \mathbb{C}^{(K-1) q \times r}$ can be expressed as

$$
\begin{aligned}
\tilde{\mathbf{G}}_{i, l} & =\tilde{\mathbf{U}}_{i, l} \tilde{\boldsymbol{\Sigma}}_{i, l} \tilde{\mathbf{V}}_{i, l}^{H} \\
& =\tilde{\mathbf{U}}_{i, l}^{H} \tilde{\boldsymbol{\Sigma}}_{i, l}\left[\begin{array}{ll}
\tilde{\mathbf{V}}_{i, l}^{\|} & \tilde{\mathbf{V}}_{i, l}^{\perp}
\end{array}\right]^{H},
\end{aligned}
$$

where, $\tilde{\mathbf{V}}_{i, l}^{\perp} \in \mathbb{C}^{r \times\left(r-\tilde{g}_{i, l}\right)}$ corresponds to the last $\left(r-\tilde{g}_{i, l}\right)$ right singular vectors that forms an orthogonal basis for the null space of $\tilde{\mathbf{G}}_{i, l}$ with rank $\tilde{g}_{i, l}=\min \{(K-1) q, r\}$. The null space when multiplied by $\tilde{\mathbf{G}}_{i, l}$ mitigates the SI. Hence the precoder at TBS $l$ can be given by

$$
\mathbf{B}_{i, l}=\tilde{\mathbf{V}}_{i, l}^{\perp} .
$$

Following the computation of precoders at each TBS, the received signal at $k$ th RBS can be expressed as

$$
\boldsymbol{r}_{k}=\sum_{l=1}^{L} \mathbf{G}_{k, l} \mathbf{B}_{k, l} \boldsymbol{s}_{k, l}+\boldsymbol{n}_{k} .
$$

Hence, the design problem to minimize the MSE between the intended information to $k$ th RBS and the actual received signal can be formulated as [9]

$$
\underset{\mathbf{Z}_{k}}{\arg \min } \mathbb{E}\left[\left\|\boldsymbol{s}_{k}-\mathbf{Z}_{k}^{H} \boldsymbol{r}_{k}\right\|_{2}^{2}\right] .
$$

By solving the above problem, the combiner $\mathbf{Z}_{k}^{H}$ at $k$ th RBS is given by

$$
\begin{aligned}
\mathbf{Z}_{k}^{H} & =\mathbb{E}\left[\boldsymbol{s}_{k} \boldsymbol{r}_{k}^{H}\right] \mathbb{E}\left[\boldsymbol{r}_{k} \boldsymbol{r}_{k}^{H}\right]^{-1} \\
& =\left[\mathbf{G}_{k, 1} \mathbf{B}_{k, 1} \mathbf{G}_{k, 2} \mathbf{B}_{k, 2} \ldots \mathbf{G}_{k, L} \mathbf{B}_{k, L}\right]^{H} \\
& \cdot\left(\sum_{l=1}^{L} \mathbf{G}_{k, l} \mathbf{B}_{k, l} \mathbf{B}_{k, l}^{H} \mathbf{G}_{k, l}^{H}+\sigma_{0}^{2} \mathbf{I}_{k}\right)^{-1} \in \mathbb{C}^{r \times L u} .
\end{aligned}
$$

\section{Number of RF Chains NeCESSARY to ACHIEVE the} HIGHEST MULTIPLEXING GAIN

In this section, we focus on to obtain a relation that determines the number of RF chains that are to be employed at each TBS and RBS to achieve the highest multiplexing gain $\rho=\sum_{(k, l)} \rho_{k, l}$, where $\rho_{k, l}$ is the multiplexing gain of link $(k, l)$. The achievable $\rho$ for a MIMO X channel with $M$ antennas at each TBS (RBS) and $N$ antennas at each RBS (TBS) is given by $\rho=\left\lfloor\frac{4 M}{3}\right\rfloor$, where $N=\left\lfloor\frac{\rho}{2}\right\rfloor[10]$. However in this work, we provide a more generalized expression for a MIMO multi-X channel that takes in to account the number of TBS and RBS employed in the network. Moreover, we start with an assumption that $\mathbf{G}_{k, l}$ has full rank. 


\section{A. $B D-B D$ scheme}

In order to exploit the left null space in (10), it is required that $q>(L-1) r$ which results in $\rho=\min \{L r, K q\}=L r$. In addition, $\rho_{k, l}$ depends on the rank of the resultant channel $\mathbf{F}_{k, l}$ (12) which is formed after decomposing the channel to $L$ non-interfering MIMO-BC sub-channels. Therefore, we can immediately write the following condition as

$$
\sum_{k=1}^{K} \sum_{l=1}^{L} \operatorname{rank}\left(\mathbf{F}_{k, l}\right)=\min \{L r, K q\} .
$$

By solving (28), the relation between the number of RF chains at each TBS and RBS can be given by

$$
q=r \cdot\left[\frac{(L-1) K+1}{K}\right] \text {. }
$$

\section{B. BD-MMSE scheme}

The BD-MMSE scheme requires $r>(K-1) q$, so that the right null space in (23) is exploited, which results in $\rho=\min \{L r, K q\}=K q$. Moreover, $\rho_{k, l}$ depends on the rank of the channel response $\mathbf{G}_{k, l}$. Therefore, we can write the following condition as

$$
\sum_{k=1}^{K} \sum_{l=1}^{L} \operatorname{rank}\left(\mathbf{G}_{k, l}\right)=\min \{L r, K q\} .
$$

Solving (30), the relation between the number of RF chains at each TBS and RBS for the scheme that decomposes the MIMO multi-X channel to $K$ non-interfering MIMO-MAC sub-channels is given as

$$
r=q \cdot\left[\frac{L(K-1)+1}{L}\right] .
$$

Note that, (29) and (31) remains valid for any design procedure that decomposes the MIMO multi-X channel to multiple MIMO-BC sub-channels and multiple MIMO-MAC subchannels.

\section{COMPLEXITY ANALYSIS}

This section studies the computational complexity involved in computing the BB precoders and combiners using the proposed schemes. The computational complexity is predominantly determined by the number of SVDs performed, whose time complexity is proportional to $O\left(\min \left\{m^{2} n, m n^{2}\right\}\right)$ for a $m \times n$ matrix. Similarly the time complexity of a matrix inverse operation is given by $O\left(n^{3}\right)$. Table. 1 shows the number of matrix operations required to compute the BB precoders and combiners for the proposed schemes.

The computational complexities involved in the computation of the precoders and combiners based on BD-BD and BDMMSE schemes are approximately given as

$$
\begin{aligned}
\text { a) } K L \cdot\left(O\left(\min \left\{q a^{2}, q^{2} a\right\}\right)^{\dagger}\right. \\
+O\left(\min \left\{(K-1)^{2}(q-a)^{2} r,(K-1)(q-a) r^{2}\right\}\right)^{\dagger} \\
\left.+O\left((r / L)^{3}\right)\right)^{(\ddagger)}, a=(L-1) r .
\end{aligned}
$$

TABLE I

TABLE SHOWING THE NUMBER OF MATRIX OPERATIONS REQUIRED TO COMPUTE THE BASEBAND PRECODERS AND COMBINERS.

\begin{tabular}{|l|c|c|c|}
\hline \multirow{2}{*}{} & \multirow{2}{*}{$\mathbf{B}_{k, l}$} & \multicolumn{2}{|c|}{$\mathbf{Z}_{k, l}$} \\
\cline { 3 - 4 } & & $\mathbf{Q}_{k, l}$ & $\mathbf{T}_{k, l}$ \\
\hline \hline BD-BD & $K L$ SVDs & $K L$ SVDs & $K L$ inverses \\
\hline BD-MMSE & $K L$ SVDs & \multicolumn{2}{|c|}{$K$ inverses } \\
\hline
\end{tabular}

$$
\begin{aligned}
& \text { b) } K \cdot\left(L \cdot O\left(\min \left\{r b^{2}, r^{2} b\right\}\right)^{\dagger}+O\left(r^{3}\right)^{\ddagger}\right) \text {, } \\
& b=(K-1) q .
\end{aligned}
$$

Note that $\dagger$ and $\ddagger$ denotes the SVD and inverse operations, respectively. Out of the two proposed schemes, the BD-MMSE scheme requires much fewer calculations compared to the BD$\mathrm{BD}$ scheme. This is due to the fact that the two stage computation of the combiner requires more computations compared to the single stage combining in BD-MMSE scheme, where a matrix inverse operation suffices to cancel the interference.

\section{Simulation Results}

In this section, we evaluate the performance of the proposed linear BB precoding and combining schemes in terms of the achieved sum rates. Equal power is assumed at all TBS, $P_{l}=$ $1, \forall l=1, \ldots, L$. Moreover, the power is uniformly distributed among all the streams transmitted from each TBS.

\section{A. Rayleigh fading channel}

We assume that the effective channel after RF precoding and combining has full rank. This means that the number of RF chains employed at each TBS and RBS, respectively are less compared to the number of individual paths $(D)$ supported by the respective channel. Therefore we model the entries of the effective channel as i.i.d. $\mathcal{C N}(0,1)$.

The number of RF chains to be employed at each TBS and RBS in a network of $L$ TBS and $K$ RBS is determined by the relation provided in Section-IV. As a reference, the performance of the optimal scheme shown in (7) is compared with the proposed sub-optimal low-complex baseband schemes for equivalent configurations of RF chains shown in Fig. 2 for $K=L=2$. The sum rate maximization problem (7) is solved for the best precoder-combiner pair that results in high sum rate with 1000 randomly initialized precoders using numerical simulations.

A single stream is transmitted between each TBS-RBS pair resulting in a total of $K L u=4$ streams which means that only the dominant singular mode is used for the transmission. The high computational complex optimal scheme which requires full knowledge of CSI at each BS (7) outperforms the lowcomplex BD-MMSE and BD-BD schemes, respectively which are based on partial CSI. When the SNR is significantly low, the BD-MMSE scheme outperforms the BD-BD scheme at low SNRs. This is due to the fact that the MBI is weak at low SNRs and the received signal is dominated by the noise. Hence, the BD-BD scheme achieves poor sum rate at low SNRs. 
However, at high SNRs, due to the high signal power, the impact of MBI and SI is more compared to the noise and hence the BD-BD scheme mitigates the aforementioned interference efficiently by projecting the desired signal on to the subspace orthogonal to the one spanned by the other RBSs channels, when BD is performed at each TBS (TBSs channel when BD is performed at each RBSs). It is believed that this projections results in significant loss of power especially at low SNRs since the noise dominates. It must be noted that in the BD$\mathrm{BD}$ scheme, $\mathrm{BD}$ is performed initially at each RBS to remove MBI, and also performed at each TBS on the intermediate channels resulting after the first stage of combining to remove SI. However, it can also be observed that both the schemes converges at high SNRs.

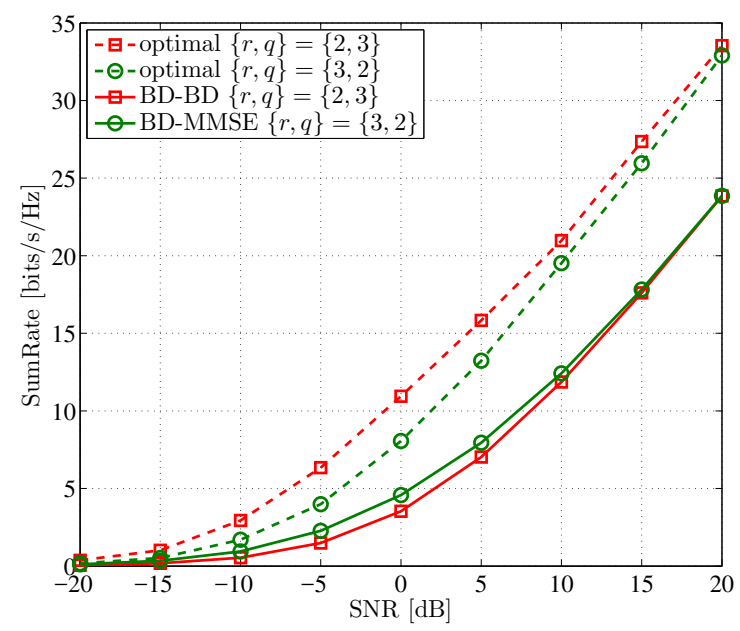

Fig. 2. Backhaul network with $k=L=2, K L u=4$ and the number of $\mathrm{RF}$ chains to be employed at each BS deduced from (29) and (31).

\section{B. mmWave channel model}

In this subsection, we assume that the effective channel that results after RF precoding and combining is rank deficient. This means that the number of RF chains employed at each BS are greater than the total number of individual paths $D$, i.e. $[r, q]>D$. Therefore, we studied the performance of the proposed schemes for two different cases using the rank deficient mmWave channel model. From the channel campaign measurements [11], [12], it has been observed that a strong line of sight (LOS) component along with few weaker multi-path components contributes to the mmWave channel. Hence from [2], we model the ray-clustered model as the contribution of single LOS component along with $D-1$ non-line of sight (NLOS) components as

$$
\mathbf{H}=\gamma\left[\alpha_{\mathrm{LOS}} \boldsymbol{a}_{r}\left(\phi_{r}^{\mathrm{LOS}}\right) \boldsymbol{a}_{t}\left(\phi_{t}^{\mathrm{LOS}}\right)+\sum_{d=1}^{D-1} \beta_{d} \boldsymbol{a}_{r}\left(\phi_{r}^{d}\right) \boldsymbol{a}_{t}\left(\phi_{t}^{d}\right)\right],
$$

where $\gamma=\sqrt{\frac{M N}{\alpha+(D-1) \beta}}$ is the normalization factor such that $\mathbb{E}\left[\|\mathbf{H}\|_{F}^{2}\right]=M N$. Here $\alpha_{\mathrm{LOS}}$ and $\beta_{d}$ are the complex gains of the LOS and NLOS components, respectively which are distributed with variance $\alpha$ and $\beta$, and $\beta=\frac{\alpha}{2}$. Moreover, $\boldsymbol{a}_{t}\left(\phi_{t}\right)$ and $\boldsymbol{a}_{r}\left(\phi_{r}\right)$ are the array response vectors (ARVs) of the TBS and RBS, respectively in the directions of azimuth $\left\{\phi_{t}, \phi_{r}\right\} \in[0,2 \pi)$. The ARVs of the uniform circular array (UCA) with $M$ antenna elements is modeled as $\boldsymbol{c}(\phi)=$ $\frac{1}{\sqrt{M}}\left[e^{j \vartheta \cos \left(\varphi-\gamma_{0}\right)}, e^{j \vartheta \cos \left(\varphi-\gamma_{1}\right)}, \ldots, e^{j \vartheta \cos \left(\varphi-\gamma_{M-1}\right)}\right]$, where $\vartheta=\frac{2 \pi r}{\lambda}$ with $r$ being the radius of the UCA, $\lambda$ is the wavelength and $\gamma_{m}=2 \pi(m-1) / M, \forall m=1,2, \ldots, M-1$.

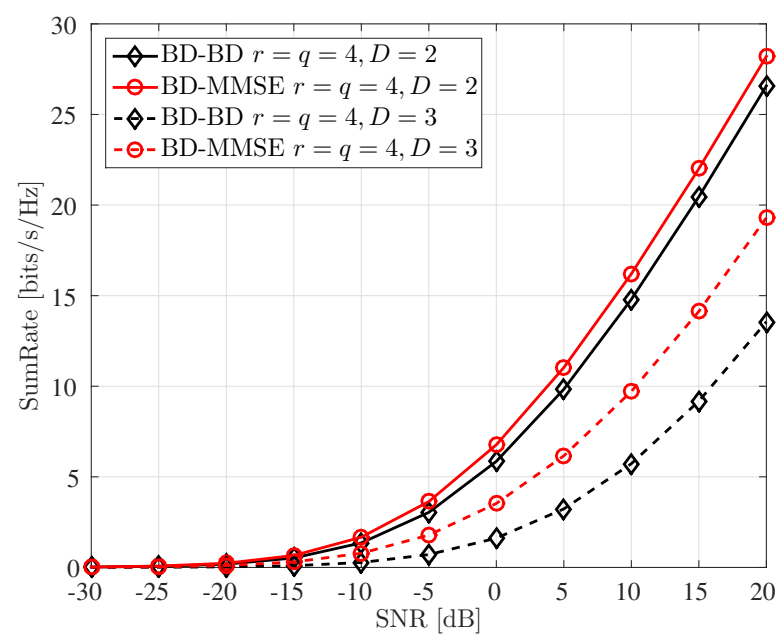

Fig. 3. Backhaul network with $K=L=2, K L u=4$ with fixed number RF chains and varying number of individual paths $D$.

In the first case, we consider $r=q=4$ at each BS and $D$ is only varied. From Fig. 3, it can be observed that the BD-MMSE scheme outperforms the BD-BD scheme for two different values of $D$. It can also be observed that when $D=3$, the sum rate achieved by both the schemes decreases compared to that when $D=2$. This is due to the fact, that only the dominant path is exploited between each TBS-RBS pair and the SVD which is used in both the schemes results in the decreasing order of singular values. In other words, when $D$ increases, the total energy of the channel is distributed among all the paths and hence the energy in the dominant path reduces. Moreover, when $D=3$, the BD-BD scheme overperforms the BD-MMSE with a huge difference. This can also be justified by the fact that the BD-BD scheme uses SVD at each RBS and TBS, respectively which results in further decrease in the sum rate compared to the BD-MMSE scheme where the SVD is used only at each TBS.

In the second case, the number of RF chains is varied and $D$ is kept fixed. From Fig. 4, it can be observed that the BD-MMSE scheme outperforms the BD-BD scheme for both $r=q=6$ and $r=q=10$, respectively. The BD-MMSE scheme has a minor difference in the achievable sum rate for 
two different configurations of RF chains, whereas the BD$\mathrm{BD}$ scheme shows a huge margin. When the number of RF chains increases, due to the normalization factor shown in (34) ( $r=M$ and $q=N$, since RF precoding and combining is not performed), the total energy of the channel increases, and hence the dominant singular value in the case of $r=q=10$ is greater compared to the case when $r=q=6$. Hence, the BDBD scheme achieves significantly low sum rate compared to the BD-MMSE scheme. From the results shown in Fig. 3 and

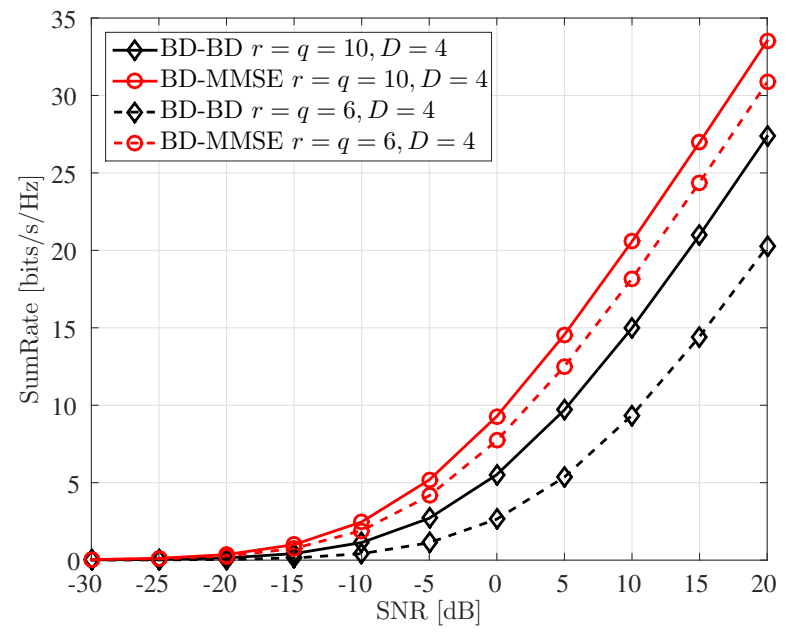

Fig. 4. Backhaul network with $K=L=2, K L u=4$ with varying number RF chains and fixed number of individual paths $D$.

Fig. 4, it can be understood $\{r, q\}$ and $D$ plays a significant role in the achievable sum rates. Moreover, the assumption of computing the BB precoders and combiners directly on the Rayleigh and mmWave channel models is also justified, since the maximum achievable sum rate in the case of hybrid precoding is bounded by the $\mathrm{BB}$ only precoding [2], [3], [6].

\section{CONCLUSION}

We have proposed novel low-complexity linear BB precoding and combining schemes for a MIMO multi-X channel operating at mmWave frequencies. The BD-BD scheme which is based on the idea of decomposing the channel to multiple non-interfering MIMO-BC sub-channels seen from each RBS, utilizes a two stage combining to align the interference. On the other hand, the interference alignment in the BD-MMSE scheme is based on decomposing the channel to multiple noninterfering MIMO-MAC channels seen from each TBS, using only a single stage combining, proved to be computationally efficient with highest achievable sum rate compared to the BDBD scheme. Numerical simulations demonstrates the superior performance of the proposed schemes. From the analysis provided, we can conclude that the low-complex BD-MMSE might be a suitable solution for the mmWave backhaul due to its high sum rate achieving capability and the single step computation of the precoders and combiners makes it worth interesting.

\section{ACKNOWLEDGMENT}

This work was supported by the European Commission in the framework of the H2020 SANSA project (Grant agreement no. 645047).

\section{REFERENCES}

[1] F. Khan and J. Pi, "Millimeter wave mobile broadband: Unleashing 3300GHz spectrum," IEEE Wireless Commun. Netw. Conf. , 2011.

[2] O. El Ayach, S. Rajagopal, S. Abu-Surra, Z. Pi, and R.W. Heath, "Spatially sparse precoding in millimeter wave MIMO systems," IEEE Transactions on Wireless Commun.ications, vol. 6, no. 1, pp. 59-631, March 2014.

[3] T. E. Bogale and L. B. Le, "Beamforming for multiuser massive MIMO systems: Digital versus hybrid analog-digital," IEEE Global Communications Conference, pp. 4066-4071, 2014.

[4] A. Alkhateeb, G. Leus, and R. W. Heath, "Limited feedback hybrid precoding for multi-user millimeter wave systems," submitted to IEEE Transactions on Wireless Communications, arXiv:1409.5162, 2014.

[5] W. Ni and X. Dong, "Hybrid Block Diagonalization for Massive Multiuser MIMO Systems," IEEE Transactions on Communications, vol. 64, no. 1, pp. 201-211, 2016.

[6] M. Kim and Y. H. Lee, "MSE-Based Hybrid RF/Baseband Processing for Millimeter-Wave Communication Systems in MIMO Interference Channels," IEEE Transactions on Vehicular Technology, vol. 64, no. 6, pp. 2714-2720, 2015.

[7] S. Boyd, and L. Vandenberghe, "Convex optimization.” Cambridge University Press, 2004.

[8] Q. H. Spencer, A.L. Swindehurst, and M. Haardt, "Zero-forcing methods for downlink spatial multiplexing in multiuser MIMO channels," IEEE Transactions on Signal Processing, vol. 52, no. 2, pp. 461-471, March 2004.

[9] T. Kailath, A. H. Sayed, and B. Hassibi, "Linear estimation," Prentice Hall New Jersey, vol.1, 2000.

[10] M. A. Maddah-Ali, A. S. Motahari, and A. K. Khandani, "Communication over MIMO X channels: Interference alignment, decomposition and performance analysis," IEEE Transactions on Information Theory, vol. 54, no. 8, pp. 3457-3470, August 2008.

[11] Y. Azar, G. N. Wong, K. Wang, R. Mayzus, J. K. Schulz, H. Zhao, F Gutierrez, D. Hwang, and T. S. Rappaport, "8 GHz propagation measurements for outdoor cellular communications using steerable beam antennas in new york city," IEEE International Conference on Communications, pp. 5143-5147, 2013.

[12] G. R. Mac Cartney and T. S. Rappaport, "73 GHz millimeter wave propagation measurements for outdoor urban mobile and backhaul communications in new york city," IEEE International Conference on Communications, pp. 4862-4867, 2014. 